\title{
FASCINATING NUMBER SEQUENCES FROM FOURTH ORDER DIFFERENCE EQUATION VIA QUATERNION ALGEBRAS
}

\author{
Asim PATra \\ Department of Mathematics \\ National Institute of Technology \\ Rourkela, Odisha, India \\ e-mail: asimp1993@gmail.com, 515ma3016@nitrkl.ac.in
}

\begin{abstract}
The balancing and Lucas-balancing numbers are solutions of second order recurrence relations. A linear combination of these numbers can also be obtained as solutions of a fourth order recurrence relation. This recurrence relation can be extended to generalized quaternion algebras. Also, the fourth order recurrence relation has application in coding theory.
\end{abstract}

Keywords: balancing numbers, Lucas-balancing numbers, quaternion algebra, Coding theory, Pell and Lucas-Pell numbers.

2010 Mathematics Subject Classification: 11B37, 11B39, 11 D09.

\section{REFERENCES}

[1] W.M. Abd-Elhameed and N.A. Zeyada, A generalization of generalized Fibonacci and generalized Pell numbers, Int. J. Math. Educ. Sci. Technol. 48 (2017) 102-107. https://doi.org/10.1080/0020739X.2016.1170900

[2] M. Basu and B. Prasad, The generalized relation among the code elements for Fibonacci coding theory, Chaos, Solitons and Fractals 41 (2009) 2517-2525. https://doi.org/10.1016/j.chaos.2008.09.030

[3] A. Behera and G.K. Panda, On the square roots of triangular numbers, Fibonacci Quarterly 37 (1999) 98-105.

[4] T.M. Cover and J.A. Thomas, Elements of Information Theory (Wiley, New York, 1991).

[5] C. Flaut and D. Savin, Quaternion algebras and generalized Fibonacci-Lucas quaternions, Adv. Appl. Clifford Algebras 25 (2015) 853-62. https://doi.org/10.1007/s00006-015-0542-0 
[6] C. Flaut and D. Savin, Some special number sequences obtained from a difference equation of degree three, Chaos, Solitons and Fractals 106 (2018) 67-71. https://doi.org/10.1016/j.chaos.2017.11.015

[7] M.E. Koroglu, I. Ozbek and I. Siap, Optimal codes from Fibonacci polynomials and secret sharing schemes, Arab J. Math. (2017) 1-12. https://doi.org/10.1007/s40065-017-0171-7

[8] G.K. Panda and P.K. Ray, Some links of balancing and cobalancing numbers with Pell and associated Pell numbers, Bull. Inst. Math. Acad. Sin. (New Series) 6 (2011) $41-72$.

[9] P.K. Ray, Some congruences for Balancing and Lucas-Balancing Numbers and their Applications, Integers 14 (2014) A8.

[10] D. Savin, About special elements in quaternion algebras over finite fields, Adv. Appl. Clifford Algebras 27 (2017) 1801-13. https://doi.org/10.1007/s00006-016-0718-2

[11] A. Stakhov, V. Massingue and A. Sluchenkov, Introduction into Fibonacci Coding and Cryptography (Osnova, Kharkov, 1999).

[12] A.P. Stakhov, Fibonacci matrices, a generalization of the Cassini formula and a new coding theory, Chaos, Solitons and Fractals 30 (2006) 56-66. https://doi.org/10.1016/j.chaos.2005.12.054

[13] J. Voight, The arithmetic of quaternion algebras, http://www.math.dartmouth.edu/jvoight/crmquat/book/ quatmodforms-041310, (2015).

Received 12 December 2019

First Revised 20 February 2020

Second Revised 8 July 2020 Accepted 11 July 2020 